\title{
Does a prenatal bacterial microbiota exist?
}

\author{
M Hornef ${ }^{1}$ and J Penders ${ }^{2}$
}

\section{THE CONCEPT OF A PRENATAL MICROBIOME}

The recent technical progress and enormous efforts to unravel the manifold interactions of the microbiota with the host's organism have provided striking and unforeseen insights. This work assigns the microbiota a central role in human health and has identified novel strategies to prevent and fight diseases in the future. One particular aspect of this work has been the early colonization of the newborn and a strong influence of maternal sources on the developing microbiota of the neonate. ${ }^{1,2}$ Birth, or more accurately rupture of the amniotic membranes that surround the embryo and separate it physically from the lumen of the uterus first exposes the neonate to the environment and is generally considered the start of the microbiota establishment. More recently, the existence of a placental microbiome, and thus maternal-fetal transmission of microorganisms and microbial colonization of the fetal organism before birth has been suggested. This commentary critically discusses the available data.

It is generally believed that the fetus in utero develops in the absence of viable microorganisms. This is consistent with the observation that cesarean sectionborn rodents can serve to generate germfree animals. ${ }^{3}$ Only with rupture of membranes and passage through the birth canal, the newborn becomes exposed to colonized maternal body surfaces and the environment initiating the establishment of the neonate's own microbiota. ${ }^{4}$ Recently, maternal-fetal transmission of commensal bacteria and the existence of a placental microbiome have been suggested. ${ }^{5-10}$ Colonization of the healthy placental and/or fetal tissue with a diverse group of metabolically active bacteria would; however, fundamentally challenge our current thinking of the development of the fetus within a sterile, protected environment. It would require new concepts to explain how bacteria can persist within host tissue but remain anatomically restricted to prevent systemic spread within the fetal organism and how preterm birth, a condition causally linked to bacterial infection of the amniotic tissue, is prevented. It would also raise important questions on the origin, composition and stability of the placental microbiome and its influence on the developing host and postnatal microbiome.

In support of the concept of a prenatal microbiome, Jiménez et al. reported on the cultural detection of low numbers of Enterococcus faecium, Staphylococcus epidermidis and Propionibacterium acnes from human cord blood samples after elective cesarean section. ${ }^{5}$ This analysis was complemented by a mouse study in which they administered a genetically labeled human E. faecium isolate orally to pregnant mice and reported detection of this strain from cultures of amniotic fluid. ${ }^{5}$ A subsequent study from the same group analyzed meconium samples of 21 healthy human neonates born by either vaginal delivery or caesarean section and cultured bacteria of the genera Staphylococcus, Enterococcus, Streptococcus, Leuconostoc, Bifidobacterium, Rothia, Bacteroides but also of the Proteobacteria Klebsiella, Enterobacter and Escherichia coli. ${ }^{6}$ Again, oral administration of the labeled E. faecium strain to pregnant mice led to the detection in meconium samples. ${ }^{6}$ They concluded the presence of "mother-to-child transmission" before birth. Three other groups described the PCR-based detection of bacteria in placental tissue. Rautava et al. detected bacterial DNA mainly from the genus Lactobacillus as well as the mostly obligate anaerobic growing genera Bifidobacterium, Bacteroides and Clostridium in 29 of 29 placental samples after elective cesarean section. ${ }^{7}$ The group of Versalovic performed a metagenomic approach on placental specimen collected under sterile conditions from 320 individuals after vaginal delivery or cesarean section and described a low-abundance microbiome including the phyla Firmicutes, Tenericutes, Proteobacteria, Bacteroides and Fusobacteria. ${ }^{8}$ A recent study by Bassols et al., examined placental tissue of 22 vaginally delivered neonates from mothers with or without gestational diabetes aseptically collected and prepared after childbirth in the delivery or operating room. ${ }^{9}$ PCR and $16 \mathrm{~S}$ rDNA sequencing revealed the presence of

${ }^{1}$ Institute of Medical Microbiology, RWTH University Hospital, Aachen, Germany and ${ }^{2}$ Department of Medical Microbiology, NUTRIM School of Nutrition and Translational Research in Metabolism \& Caphri School for Public Health and Primary Care, Maastricht University Medical Centre, Maastricht, The Netherlands. Correspondence: MW Hornef (mhornef@ukaachen.de) 
Proteobacteria, Bacteroidetes, Firmicutes, and Actinobacteria. ${ }^{9}$ Placental tissue of women with gestational diabetes exhibited a distinct microbiota profile which was amongst others characterized by a lower relative abundance in the Pseudomonadales order and Acinetobacter genus and an increased abundance in the Lachnospiraceae, Coriobacteriaceae and Bradyrizobiaceae families and the genera Escherichia and Parabacteroides. ${ }^{9}$ Both PCR- and culture-based methods were used by the group of Salminen to examine placental tissue and amniotic fluid of 15 electively cesarean section delivered human newborns. 16S rDNA sequencing detected a "low-richness, low-diversity" bacterial composition with a predominance of Proteobacteria. ${ }^{10}$ Culture mainly revealed bacteria of the genera Staphylococcus and Propionibacterium (but notably not the known human pathogen S. aureus or any Proteobacteria). They concluded the existence of "microbial transfer at the foeto-maternal interface" and the presence of a "fetal microbiota". ${ }^{10}$ A recent PCR-based study on early bacterial airway colonization demonstrated the presence of a diverse and distinct lung microbiome at birth and also hypothesized fetal microbiota acquisition to explain their findings. ${ }^{11}$

Although maternal-fetal transfer of commensal bacteria would reduce the environmental influence and could thereby help to explain stable transmission of the microbiota over many generations, the currently available data supporting the concept of a placental or fetal microbiota at this point are highly questionable for the following reasons.

\section{DISCUSSION OF THE AVAILABLE EVIDENCE}

The detection of DNA fragments by PCR using 25 or more cycles is extremely sensitive. Minute amounts of even heavily damaged DNA can already be detected. Deep sequencing with short reads as typically employed for NGS then reveals also very rare DNA molecules. The problem is that DNA of microbial origin is ubiquitous in our environment and contaminates our laboratory equipment including plastic tubes, forceps or pipet tips and (even commercial) reagents such as spin columns or enzymes representing a source for false positive results. This problem is well known to medical microbiologists or forensic scientists who are frequently confronted with the situation to judge on the value and meaning of a positive PCR signal. Of note, this problem can almost be neglected when studying densely populated ecological niches such as the enteric microbiota but becomes a critical issue when studying samples with low bacterial density. Therefore, extensive technical precautions during sample acquisition and processing, the implementation of adequate internal controls at all steps during sample preparation and analysis and a critical interpretation of the results are required. Previous studies did not incorporate internal controls or only at some stages during the experimental procedures (e.g., at DNA isolation and sequencing) but not at sample collection. ${ }^{8,9}$ A bacterial composition similar to that found in the environment or the human oral or skin microbiota should raise doubts on the origin. One study detected a similar microbial density and bacterial community structure in placental specimen and air swabs from the processing room. ${ }^{12}$ Two other studies highlighted the similarity of the bacterial spectrum detected in placental tissue with the human oral microbiome or the skin microbiota., Another way to reveal a contamination bias is to use different purification methods with the same samples. A defined bacterial community is expected to display a similar composition irrespective of the method and reagents used. A recent comparative $16 \mathrm{~S}$ rDNA based analysis of placental samples with contamination controls employed two different purification methods and failed to provide evidence for a defined placental spectrum of bacterial DNA. ${ }^{12}$ Finally, a consistent detection of bacterial DNA and a certain degree of similarity in the bacterial composition between different studies, i.e., a "core" microbiome would be postulated. However, one study reported that all 16 amniotic fluid samples obtained from healthy pregnant females remained negative by PCR and culture and significant differences in the main taxa of the placental microbiome were described in three other reports. ${ }^{8-10,13}$

Additionally, transient bacteremia, i.e., the presence of viable bacteria in blood is occasionally observed in the healthy host. For example, bacteria can be cultured from blood samples after dental hygiene. ${ }^{14}$ Similarly, mechanical manipulation of heavily colonized genital body surfaces as it occurs during vaginal delivery or the abdominal skin incision during cesarean section is expected to facilitate bacterial entry into the maternal bloodstream. ${ }^{15}$ Although bacteria are rapidly eliminated by serumderived or cellular host defense mechanisms in the healthy host, this can explain the occasional detection of viable bacteria at low-abundance in blood and tissue samples including the heavily perfused placental tissue. It can also explain the detection of bacterial DNA at sterile body sites. Although sensitive to nucleases, DNA remains detectable in blood for a certain time and could reach the placental tissue with the bloodstream or intracellularly in maternal phagocytic migratory cells.

Moreover, the detection of DNA by definition is unable to prove the existence of a bona fide microbiota. The word microbiota composed of the two greek words $\mu \iota \kappa \rho о \sigma$ (small) and $\beta 10 \sigma$ (life) describes a community of microorganisms that colonize an ecological niche and refers to the presence of viable, proliferating and metabolically active microorganisms. The detection of bacterial DNA is not necessarily equivalent to the presence of viable bacteria but might be derived from microbial remnants at other body sites or the environment. $^{7-10}$ Interestingly, one report described the detection of bacteria by light microscopy following Giemsa, Gram, and Brown-Hopps staining of paraffin tissue sections of the basal plate of the placenta in $27 \%$ of pregnant women without a significant link to chorioamnionitis or preterm birth. ${ }^{16}$ Strikingly, the authors highlighted the intracellular localization of the detected bacteria reminiscent of invasive infection rather than colonization. ${ }^{16}$ Similar to the 
PCR results, this finding awaits confirmation and the demonstration of bacterial viability and diversity within individual samples.

Many bacteria can multiply very rapidly. For example, E. coli can divide approximately every $20 \mathrm{~min}$ when supplied with sufficient nutrients. The contact of the neonate's nose, mouth, and anus with densely colonized maternal body surfaces during passage through the birth canal thus rapidly leads to colonized mucosal surfaces. Sample material acquired through the mouth or nose and meconium released through the anus therefore does not represent a suitable sample material to proof the presence of a fetal microbiota. ${ }^{6}$ Any contact of sample material with maternal body surfaces will inevitably contaminate the material.

The placenta attaches intimately to the uterus surface to facilitate efficient exchange of oxygen and nutrients between the maternal and fetal circulation. It does not exhibit a luminal space or internal mucosal surface and thus colonizing microorganisms would have to persist within host tissue and survive exposure to oxygen and humoral as well as cellular host defense mechanisms. The bacteria suggested to contribute to a placental microbiota such as members of the genera Propionibacterium, Enterococcus, Lactobacillus, Bacteroides, Clostridium and coagulase-negative Staphylococci (i.e., staphylococcal species other than $S$. aureus) are typical commensal bacteria and colonize the adult skin or intestinal tract at high density. Infections with these bacteria are rarely observed in the healthy host but associated with foreign bodies (i.e., intravenous catheters) or strong immunosuppression (i.e., after bone marrow transplantation). It is questionable whether these bacteria are able to resist the cellular and serum-derived antibacterial defense mechanisms and survive for prolonged time in healthy tissue. Other reported microorganisms such as Staphylococcus aureus and Escherichia coli are known to resist the innate host defense. Yet they typically cause pyogenic infections and would therefore be expected to induce a strong local inflammatory response in placental tissue. Such an inflammatory response has not been described and would be hard to reconcile with an ongoing pregnancy. Chorioamnionitis, i.e., an inflammation of the amniotic sac and placental tissue is the most common known cause of preterm delivery. ${ }^{17}$ The bacteria associated with chorioamnionitis and preterm birth by cultural methods belong to the genera Ureaplasma, Mycoplasma, Fusobacterium, Gardnerella, and Bacteroides. ${ }^{13}$ This spectrum of cultured bacteria differs from what has been detected by PCR-based methods. ${ }^{13}$ The reason is unclear but may again be the fastidious growth or non-culturable state of individual bacterial species or DNA contamination. Some specialized bacteria as well as prokaryote endosymbionts have adopted lifestyles that facilitate long-term persistence within host tissue with only minor or no inflammatory response. Other bacterial species can enter a stage of reduced metabolic and proliferative activity referred to as dormant or "viable but not culturable", which might facilitate their prolonged presence in tissue. The detection of individual bacteria, however, would not be referred to as a microbiota but endosymbiosis or chronic infection although the meaning of these terms may at some point overlap.

\section{CONCLUSIONS AND FUTURE RECOMMENDATIONS}

Altogether, there is no compelling evidence for the existence of a universal mammalian placental or fetal microbiota to date. The available results, however, warrant further analysis. Larger sample sizes, the simultaneous use of different detection methods, the elimination of extracellular DNA prior to molecular microbial profiling and rigorous controls for reagents and equipment at all steps during sample processing and analysis are required to establish a consistent bacterial profile. ${ }^{18}$ The determination of the relative abundance of bacterial groups should be preceded by an absolute quantification of the bacterial load in samples and controls (e.g., using a quantitative eubacterial PCR). Negative controls should be implemented at all steps during the experimental procedure and the addition of prespecified and quantified bacterial mock communities to the examined samples will help to reveal biases and identify batch effects. ${ }^{12,19}$ Subsequently, the demonstration of metabolically active and proliferating diverse bacteria within the placental or fetal tissue will be required to prove the existence of a viable, diverse and unique bacterial community that merits the term microbiota. Although microbiota research has allowed unexpected and exciting insights during the last years that undoubtedly will change our understanding of the host-microbial relationship, we should keep a healthy dose of scepticism and critically view the current evidence on the existence of a prenatal bacterial microbiota.

Importantly, there is no need to postulate a placental or fetal microbiota to explain the influence of microorganisms or microbial components on cellular functions of the fetal tissue. Several reports have demonstrated systemic effects of the microbiota presumably through the dissemination of microbial constituents. The group of Jeff Weiser was the first to report an influence of gut microbiota-derived peptidoglycan on neutrophil function in the bone marrow. ${ }^{20}$ Exposure of liver tissue to bacterial DNA and lipopolysaccharide derived from the enteric microbiota and transported through the portal vein was shown to promote non-alcoholic fatty liver disease by the Flavell group. ${ }^{21}$ Pulendran and coworkers highlighted the systemic effect of bacterial flagellin from gut microbiota as a natural adjuvant during intramuscular vaccine administration. $^{22}$ More recently, the group of Andrew MacPherson demonstrated antibody-dependent spread of microbial constituents to the fetal organism during pregnancy. ${ }^{23}$

\section{ACKNOWLEDGMENTS}

This work has been supported by the Deutsche Forschungsgemeinschaft (Ho2236/8-1 and Priority Program 1656 to M.W.H).

\section{DISCLOSURE}

The authors declare no conflict of interest.

c 2017 Society for Mucosal Immunology 


\section{REFERENCES}

1. van Best, N., Hornef, M.W., Savelkoul, P.H. \& Penders, J. On the origin of species: factors shaping the establishment of infant's gut microbiota. Birth Defects Res. C Embryo Today 105, 240-251 (2015).

2. Tamburini, S., Shen, N., Wu, H.C. \& Clemente, J.C. The microbiome in early life: implications for health outcomes. Nat. Med. 22, 713-722 (2016).

3. Pleasants, J.R. Rearing germfree cesareanborn rats, mice, and rabbits through weaning. Ann. N Y Acad. Sci. 78, 116-126 (1959).

4. Dominguez-Bello, M.G. et al. Delivery mode shapes the acquisition and structure of the initial microbiota across multiple body habitats in newborns. Proc. Natl Acad. Sci. USA 107, 11971-1195 (2010).

5. Jiménez, E. et al. Isolation of commensal bacteria from umbilical cord blood of healthy neonates born by cesarean section. Curr. Microbiol. 51, 270-274 (2005).

6. Jiménez, E. et al. Is meconium from healthy newborns actually sterile? Res. Microbiol. 159, 187-193 (2008).

7. Rautava, S., Kainonen, E., Salminen, S. \& Isolauri, E. Maternal probiotic supplementation during pregnancy and breast-feeding reduces the risk of eczema in the infant. J. Allergy Clin. Immunol. 130, 1355-1360 (2012).

8. Aagaard, K., Ma, J., Antony, K.M., Ganu, R., Petrosino, J. \& Versalovic, J. The placenta harbors a unique microbiome. Sci. Transl. Med. 6, 237ra65 (2014).

9. Bassols, J. et al. Gestational diabetes is associated with changes in placental microbiota and microbiome. Pediatr. Res. 80, 777-784 (2016).

10. Collado, M.C., Rautava, S., Aakko, J., Isolauri, E. \& Salminen, S. Human gut colonisation may be initiated in utero by distinct microbial communities in the placenta and amniotic fluid. Sci. Rep. 6, 23129 (2016).

11. Lal, C.V. et al. The airway microbiome at birth. Sci. Rep. 6, 31023 (2016).

12. Lauder, A.P. et al. Comparison of placenta samples with contamination controls does not provide evidence for a distinct placenta microbiota. Microbiome 4, 29 (2016).

13. Han, Y.W., Shen, T., Chung, P., Buhimschi, I.A. \& Buhimschi, C.S. Uncultivated bacteria as etiologic agents of intra-amniotic inflammation leading to preterm birth. J. Clin. Microbiol. 47, 38-47 (2009).

14. Bhanji, S., Williams, B., Sheller, B., Elwood, T. \& Mancl, L. Transient bacteremia induced by tooth-brushing a comparison of the Sonicare toothbrush with a conventional toothbrush. Pediatr. Dent. 24, 295-299 (2002).

15. Smaill, F.M. \& Grivell, R.M. Antibiotic prophylaxis versus no prophylaxis for preventing infection after cesarean section. Cochrane Database Syst. Rev. 10, CD007482 (2014).
16. Stout, M.J. et al. Identification of intracellular bacteria in the basal plate of the human placenta in term and preterm gestations. Am. J. Obstet. Gynecol 208, 226.e1-7 (2013).

17. Goldenberg, R.L., Hauth, J.C. \& Andrews, W.W. Intrauterine infection and preterm delivery. N. Engl. J. Med. 342, 1500-1507 (2000).

18. Tantikachornkiat, M., Sakakibara, S., Neuner, M. \& Durall, D.M. The use of propidium monoazide in conjunction with $\mathrm{APCR}$ and Illumina sequencing to identify and quantify live yeasts and bacteria. Int. J. Food Microbiol. 234, 53-59 (2016).

19. Brooks, J.P. et al. The truth about metagenomics: quantifying and counteracting bias in $16 \mathrm{~S}$ rRNA studies. BMC Microbiol. 15, 66 (2015).

20. Clarke, T.B., Davis, K.M., Lysenko, E.S., Zhou, A.Y., Yu, Y. \& Weiser, J.N. Recognition of peptidoglycan from the microbiota by Nod1 enhances systemic innate immunity. Nat. Med. 16, 228-231 (2010).

21. Henao-Mejia, J. et al. Inflammasome-mediated dysbiosis regulates progression of NAFLD and obesity. Nature 482, 179-185 (2012).

22. Oh, J.Z. et al. TLR5-mediated sensing of gut microbiota is necessary for antibody responses to seasonal influenza vaccination. Immunity 41, 478-492 (2014).

23. Gomez de Agüero, M. et al. The maternal microbiota drives early postnatal innate immune development. Science 351, 1296-1302 (2016). 